\title{
PAPERS
}

\section{Cost effectiveness of treatment for gastro-oesophageal reflux disease in clinical practice: a clinical database analysis}

\author{
A Eggleston, A Wigerinck, S Huijghebaert, D Dubois, A Haycox
}

\begin{abstract}
Background-Previous evaluations of the cost effectiveness of antireflux medication used in gastro-oesophageal reflux disease (GORD) have been based on results obtained in controlled clinical trials. Unfortunately such an approach does not necessarily identify the therapeutic option which provides the greatest benefit from available resources in real life situations. To make an informed choice requires a recognition that the costs and benefits of therapy in practice may differ from those identified in trials.

Aims-To evaluate, based on a retrospective prescription database analysis, the cost effectiveness of alternative treatment options for patients with uncomplicated GORD. The analysis assesses health service resource use during the first six months of treatment in three groups of patients initially prescribed cisapride (CIS), ranitidine (RAN), or omeprazole (OME).

Methods-The MediPlus ${ }^{\text {TM }}$ UK database was used to identify all health care resources consumed by patients in the three treatment groups during their first six months of treatment. Patients with more complicated GORD, as indicated by initial referral to a specialist or outpatient hospital visit $(<13 \%)$, were excluded from the analysis.
\end{abstract}

International

Outcomes Research, Janssen Research

Foundation

A Eggleston

D Dubois

Department of Internal Medicine, Janssen Research

Foundation, Beerse, Belgium

A Wigerinck

Pharmaceutical Sciences, La Hulpe, Belgium

S Huijghebaert

Department of Pharmacology and Therapeutics, University of Liverpool, UK A Haycox

Correspondence to: Dr D Dubois, Janssen Research Foundation, Turnhoutseweg 30, B-2340 Beerse, Belgium.

Accepted for publication 16 July 1997

Results-The average cost per patient for the initial six months of treatment for CIS, RAN, and OME based therapies was $£ 136, £ 177$, and $£ 189$ per patient, respectively. A major element underlying this cost variation was the acquisition cost and quantity of antireflux medication required by patients. The average number of one month equivalent prescriptions consumed during this six month period was 1.85 (CIS), 2.57 (RAN), and 2.96 (OME) with associated costs of $£ 49$ (CIS), $£ 67$ (RAN), and $£ 105$ (OME). Antacid and alginate/antacid use was higher in the CIS and RAN groups (about 1.0 antacid prescription per patient versus 0.4 for $O M E$ ), but their contribution to the total cost per patient was less than $2 \%$. The number of general practitioner consultations over the six month period for each treatment group was 2.4 (CIS), 2.9 (RAN), and 2.6 (OME) with associated costs of $£ 60.31$ (CIS), $£ 73.06$ (RAN), and $£ 65.52$ (OME). The average number of non-drug interventions (referrals, outpatient visits, endoscopies, barium meals, or $x$ rays) was 0.34 in the RAN group compared with less than 0.2 in the CIS and OME groups. The costs associated with such interventions were $£ 23.80$ (RAN), $£ 9.60$ (CIS), and $£ 11.10$ (OME) per patient.

Conclusion-The data indicate that the "step up" approach, starting with a prokinetic or $\mathrm{H}_{2}$ receptor antagonist, represents the most cost effective initial therapeutic strategy for a primary care physician to adopt when faced with a patient with first diagnosis of uncomplicated GORD.

(Gut 1998;42:13-16)

Keywords: cisapride; ranitidine; omeprazole; gastrooesophageal reflux disease; cost; outcomes research
Over the past decade health care expenditure for gastrointestinal disease has risen sharply throughout the world with the introduction of new drugs and the increased use of diagnostic interventions all contributing significantly to the growing financial burden being imposed on the health services. This has led to a situation in which approximately $£ 250$ million is spent every year by primary care physicians in the UK alone on drug treatment to control the symptoms of upper gastrointestinal disease. Despite this level of expenditure (equivalent to $10 \%$ of the NHS drug budget) primary care physicians still face a growing level of demand generated by this pathology. Within this general therapeutic area the problems presented by patients with gastro-oesophageal reflux disease (GORD) are likely to be particularly troublesome given their tendency to require long term support. In such circumstances not only do such patients represent a continuous long term drain on health care resources but treatment also frequently fails to provide a satisfactory solution for either the patient or the doctor.

The extent of the therapeutic burden imposed by GORD on both patients and physicians is reflected in the intense evaluative focus placed 
on this therapeutic area which includes a number of previous economic evaluations of antireflux treatments. ${ }^{1-6}$ The two main therapeutic strategies analysed in previous studies are the "step up" and the "step down" approaches to initial treatment for uncomplicated GORD. The step up approach follows the principle of applying the minimum pharmacological force necessary to achieve a stated therapeutic objective. This approach targets more powerful and costly interventions selectively towards patients in which less radical intervention has met with limited success and therefore patients have a proven therapeutic need for such intensive treatment. In contrast, the step down approach proposes that patients should initially be treated with a proton pump inhibitor with therapy only being stepped down to less intensive interventions in strictly defined circumstances. The principal problem inherent in this approach is the universal application of a powerful and costly drug in patients in whom less intensive interventions may have been adequate and have not previously been proven to be ineffective.

One shortcoming exhibited by all previous evaluations is their focus on costs and benefits derived from results obtained in the rarefied atmosphere of clinical trials rather than from observations of drug use in daily clinical practice. In particular the selection bias inherent in clinical trials may render any conclusions drawn to be of limited relevance to the mainstream GORD population encountered by physicians in primary care. The costs and benefits of therapeutic strategies in the real world will also be affected by less rigorous monitoring, variations in patient compliance, provision of therapy by non-specialists, and the reduced availability of specialist diagnostic equipment to support clinical decision making. In recognition of such factors alternative data sources and analytical techniques have been developed to augment the results obtained in clinical trials and to assist in shifting the evaluative focus from short term clinical efficacy to long term clinical effectiveness. Modelling techniques such as Markov chain analyses are becoming increasingly utilised with their underlying assumptions being based on systematic reviews of the literature or expert clinical opinion. In the absence of a rigorous systematic review of therapeutic strategies for non-ulcer dyspepsia (which is currently being commissioned by the NHS) evaluation of real world treatment strategies must rely on observational studies; the largest observational studies currently available are those contained in medical databases. The clinical database chosen for analysis (MediPlus ${ }^{\mathrm{TM}} \mathrm{UK}$ ) contains the treatment histories of more than one million patients and facilitates the detailed analysis of diagnostic procedures, prescribing patterns, specialist referrals, and other health care interventions in any therapeutic area. The database therefore enables an analysis to be undertaken of the cost and effectiveness of treatment actually provided to patients with uncomplicated GORD in primary care rather than in the artificial environment generated in clinical trials.
Evaluation of the relative cost effectiveness of alternative therapeutic options in the treatment of GORD requires the cost imposed on the health care system to be analysed in relation to the outcomes achieved. Previous work ${ }^{7}$ evaluating the comparative clinical outcome of drug treatment for uncomplicated GORD in standard practice has identified equivalence in therapeutic outcome between cisapride, ranitidine, and omeprazole based therapies. Given such therapeutic equivalence it is appropriate for this analysis to concentrate on identifying the strategy that achieves this common outcome with the lowest consumption of scarce health care resources.

\section{Methods}

SAMPLE SELECTION

The MediPlus ${ }^{\mathrm{TM}}$ UK database was searched to identify patients presenting to primary care physicians during 1995 with a first episode of uncomplicated GORD. Patients with a diagnosis of dysphagia or complicated GORD or whose condition was sufficiently complex to require an immediate specialist referral were excluded from the analysis. Such strict exclusion criteria were felt to be necessary to minimise any potential selection bias when categorising patients into treatment groups based on their initial antireflux medication (cisapride, ranitidine, or omeprazole). It could be argued that primary care physicians selectively treat patients exhibiting more severe GORD with omeprazole and hence it becomes necessary to standardise the patient population, as far as possible, to take account of any such selection bias.

The case records for a total of 257 patients were evaluated; 80 patients were initially treated with cisapride, 90 with ranitidine, and 87 with omeprazole. The three patient groups were analysed and found to be comparable with respect to age, gender, and comorbidity with other forms of gastrointestinal disease. ${ }^{7}$

IDENTIFICATION, MEASUREMENT, AND VALUATION OF RESOURCE USE

All health care resources consumed by patients during the six months following initial diagnosis were identified and analysed in detail. Every prescription issued for each patient was analysed to assess the number and strength of tablets and hence the associated cost. In addition the number of consultations with primary care physicians, referrals to specialist, and episodes of hospitalisation were recorded and an appropriate cost applied. Finally, the number and nature of diagnostic tests required by each patient were assessed and costed. Drug prices were based on NHS prices contained in the British National Formulary. The costs associated with primary care consultations, referrals, and investigations were based on the charges incurred by fundholding practices using the facilities of the Royal Berkshire Hospital in Reading. All costs are expressed in pounds sterling and are provided at a 1996 price base.

\section{Results}

Table 1 summarises the results of the analysis. The average cost of health care resources con- 
Table 1 Resource use and cost in the treatment of uncomplicated GORD

\begin{tabular}{|c|c|c|c|c|c|c|}
\hline & \multicolumn{6}{|c|}{ Cost/100 patients } \\
\hline & \multicolumn{2}{|l|}{ CIS } & \multicolumn{2}{|c|}{$R A N$} & \multicolumn{2}{|c|}{$O M E$} \\
\hline & No & $\operatorname{Cost}(£ / \$)$ & No & $\operatorname{Cost}(£ / \$)$ & No & $\operatorname{Cost}(£ / \$)$ \\
\hline \multicolumn{7}{|c|}{ Drug costs: comparator drugs (one month equivalent) } \\
\hline Cisapride $10 \mathrm{mg}$ (84 tablets) & 185 & $4875 / 7703$ & 1 & $21 / 33$ & 1 & $20 / 32$ \\
\hline Ranitidine 150 mg (56 tablets) & 2 & $65 / 103$ & 257 & $6673 / 10543$ & 11 & $299 / 472$ \\
\hline Omeprazole $20 \mathrm{mg}$ (28 tablets) & 34 & $1219 / 1926$ & 23 & $808 / 1277$ & 296 & $10507 / 16601$ \\
\hline Cimetidine/other prokinetics & 6 & $92 / 145$ & 4 & $113 / 179$ & 7 & $194 / 307$ \\
\hline Alginate/antacids & 96 & $254 / 401$ & 104 & $358 / 566$ & 39 & $104 / 164$ \\
\hline Other medication & 7 & $33 / 52$ & 10 & $6 / 9$ & 11 & $33 / 52$ \\
\hline \multicolumn{7}{|l|}{ Non-drug costs } \\
\hline GP consultations & 241 & $6031 / 9529$ & 292 & $7306 / 11543$ & 262 & $6552 / 10352$ \\
\hline Specialist referrals/outpatient visits & 11 & $788 / 1245$ & 23 & $1633 / 2580$ & 10 & $724 / 1144$ \\
\hline Diagnostic tests & 3.5 & $172 / 272$ & 10 & $743 / 1174$ & 6 & $389 / 615$ \\
\hline Total cost (all resources) & & $13611 / 21505$ & & $17708 / 27979$ & & $18939 / 29923$ \\
\hline
\end{tabular}

sumed by patients from each of the three groups during this initial six month period was $£ 136$ (CIS), £177 (RAN), and £189 (OME). The average number of prescriptions for antireflux medication provided to patients was 1.85 (CIS), 2.57 (RAN), and 2.96 (OME) with associated costs of $£ 49$ (CIS), $£ 67$ (RAN), and $£ 105$ (OME). The use of other medications was limited both in terms of amount and cost, with antacid use being higher in the CIS and RAN groups but in each group the cost was less than $2 \%$ of the total cost. The average number of primary care consultations per patient over the initial six month period analysed was 2.4 (CIS), 2.9 (RAN), and 2.6 (OME) with an estimated cost associated with these consultations of $£ 60.31$ (CIS), $£ 73.06$ (RAN), and $£ 65.52$ (OME). The number of specialist referrals and diagnostic procedures was significantly higher in the RAN group (0.34) than in the CIS (0.15) and OME (0.16) groups. The estimated cost per patient of such referrals and procedures was $£ 23.80$ (RAN) compared with $£ 9.60$ (CIS) and $£ 11.10$ (OME) which constitutes $13 \%$ of total cost in the RAN group compared to $7 \%$ of total cost in the CIS group and $6 \%$ of total cost in the OME group.

The cost of interventions in the OME group was lower than in the RAN group, probably because of the better symptom control obtained with OME, leading to repeated prescriptions rather than new consultations and/or referrals. The higher cost of non-drug interventions in the RAN group in comparison to the OME group is probably explained by its lower potency to suppress acid secretion leading to more patients reconsulting the physician. This higher cost was offset by the lower cost of the medication itself. The lower cost of CIS intervention versus $\mathrm{OME}$ can be explained by the lower number of relapses ${ }^{7}$ and the fact that in cases of insufficient response patients are usually switched to an $\mathrm{H}_{2}$ receptor antagonist or $\mathrm{OME}$ before being referred for investigation. It is interesting to note that only one out of eight patients was actually seen for endoscopy or an outpatient hospital visit during this period. This indicates that the vast majority of initial treatments are given by general practitioners without prior investigation. Table 1 provides full details of the results of the analysis.

\section{Discussion}

The step down approach to treatment of GORD has been acclaimed as being the most cost effective treatment option. ${ }^{8}$ Such a claim is not supported by the results of this study. Conversely the analysis emphasises the comparative cost effectiveness of the step up approach to treatment of GORD given that initial treatment with omeprazole exhibited the highest cost of treatment. This result is almost entirely due to the higher usage and acquisition cost of omeprazole given that the costs of other drug and non-drug interventions were comparable to, or even lower than those in the CIS and RAN groups.

In interpreting the results obtained it is necessary to recognise the possibility of a selection bias with patients experiencing more severe heartburn on their first presentation being more likely to be treated with omeprazole. However, it is known that severity of symptoms is not necessarily indicative of severity of underlying disease. In addition, given that patients with refractory or complicated GORD were excluded, the extent of any potential selection bias has been minimised as far as possible. If more severely affected patients were selectively treated with omeprazole, one would expect to see increased non-drug resource use in this group. This did not occur.

Many factors are likely to underpin the primary care physician's choice of therapy and further research is required concerning this. Equally it is important to gain a greater understanding of the factors underlying a primary care physician's decision to cease therapy. One of the fundamental factors underlying the increase in expenditure on treatment for GORD is the rapidly growing number of patients becoming chronic or long term users of antireflux medication. Such chronic dependence on drug therapy appears to be a particular problem with regard to prescribing of omeprazole and represents a further argument in favour of the step up approach aimed at initially targeting prescribing of this powerful drug on a highly selected patient group.

\section{Conclusion}

Information on the comparative cost effectiveness of therapeutic interventions in clinical practice is essential if clinicians are to make the best use of existing resources to provide patient 
care. The two predominant cost elements underlying initial treatment at the primary care level for uncomplicated GORD are primary care consultations (35-44\% of total costs) and antireflux medication ( $42-45 \%$ of total costs). The results provide strong support for a step up approach to the initial management of patients with uncomplicated GORD of the primary care level - that is, reserving more potent antireflux medication for cases in which adequate symptom control has not been achieved. The results of this analysis, however, must be interpreted as being indicative rather than conclusive as more detailed analysis is required of the costs and benefits arising from different options in the therapeutic management of GORD at the primary care level.

1 Bate CM, Richardson PDI. Symptomatic assessment and cost effectiveness of treatments for reflux oesophagitis: comparisons of omeprazole and histamine $\mathrm{H}_{2}$-receptor antagonists. Br f Med Econ 1992;2:37-48.

2 Bate CM, Richardson PDI. A one year model for the cost effectiveness of treating reflux oesophagitis. Br f Med Econ 1992;2:5-11.

3 Bate CM, Hungin APS, Wilcock C, Turbitt ML, Richardson PDI. Cost effective management of gastroesophageal roflux disease in general practice. $B r \quad f$ Med Econ 1993;6:81-90.

4 Hillman AL, Bloom BS, Fendrick AM, Schwartz JS. Cost and quality effects of alternative treatments for persistent gastro-esophageal reflux disease. Arch Intern Med 1992; 152:1467-72.

5 Hillman AL. Economic analysis of alternative treatments for persistent gastro-oesphageal reflux disease. Scand 7 Gastroenterol 1994;29(suppl 201):98-102.

6 Jönsson B. Economic evaluation of new medical technology. Scand $\mathcal{F}$ Gastroenterol 1994;29(suppl 201):89-90.

7 Eggleston A, Wigerinck A, Haycox A. Outcome research in GORD: retrospective analysis of prescription data (MediPlus ${ }^{\mathbb{B}} \mathrm{UK}$ ) on cisapride (CIS), ranitidine (RAN) and omeprazole (OME) [abstract]. Gut 1996;39(suppl 3): A106.

8 Freston JW, Malagelada JR, Petersen H, McCloy RF. Critical issues in the management of gastroesophageal reflux disease. Eur f Gastroenterol Hepatol 1995; 7:577-86. 